\title{
BMJ Open Prevalence of left atrial appendage thrombus in patients with acute ischaemic stroke and sinus rhythm: a cross-sectional study
}

\author{
Huan Thanh Nguyen (D , 1,2 Hai Van Be Nguyen, ${ }^{2}$ Huy Quang Nguyen, ${ }^{2}$ \\ Hung Quoc Le $\mathrm{C}^{2}$
}

To cite: Nguyen $\mathrm{HT}$, Nguyen HVB, Nguyen $\mathrm{HQ}$, et al. Prevalence of left atrial appendage thrombus in patients with acute ischaemic stroke and sinus rhythm:

a cross-sectional study. BMJ Open 2021;11:e051563. doi:10.1136/

bmjopen-2021-051563

- Prepublication history and additional supplemental material for this paper are available online. To view these files, please visit the journal online (http://dx.doi.org/10.1136/ bmjopen-2021-051563)

Received 23 March 2021 Accepted 01 December 2021

A) Check for updates

(C) Author(s) (or their employer(s)) 2021. Re-use permitted under CC BY-NC. No commercial re-use. See rights and permissions. Published by BMJ.

${ }^{1}$ Department of Geriatrics and Gerontology, University of Medicine and Pharmacy at Ho Chi Minh city, Ho Chi Minh City, Vietnam

${ }^{2}$ Department of Cardiology, Thong Nhat Hospital, Ho Chi Minh, Vietnam

Correspondence to Dr Huan Thanh Nguyen; huannguyen@ump.edu.vn

\section{ABSTRACT}

Objective Thrombi originating in the left atrial appendage (LAA) mainly form because of atrial fibrillation (AF) and are a known cause of cardioembolic stroke. We aimed to investigate the prevalence of LAA thrombus in patients with acute ischaemic stroke (AIS) and sinus rhythm on 12-lead ECG.

Methods From June 2019 to February 2021, we conducted a cross-sectional study wherein we performed transoesophageal echocardiography (TEE) in patients with AIS and sinus rhythm on 12-lead ECG who were referred for detection of LAA thrombus. After TEE, all patients underwent 24-hour ECG monitoring to screen for paroxysmal AF. Predictors of LAA thrombus were determined using logistic regression analysis.

Results Overall, 223 patients (age: $66.2 \pm 11.3$ years, men: $61.4 \%$ ) were included in the study. LAA thrombus was detected in 15 patients $(6.7 \%)$. Paroxysmal AF was detected in 14 of the 15 patients during 24-hour ECG monitoring. Compared with the non-thrombus group, the thrombus group had a statistically significant higher rate of spontaneous echo contrast (SEC), longer LAA, lower peak LAA emptying velocity and predominantly bilateral stroke. In the adjusted model, the presence of SEC increased the probability of LAA thrombus (OR $9.04 ; 95 \% \mathrm{Cl} 2.12$ to 38.54; $p=0.003$ ).

Conclusions In patients with AIS and sinus rhythm on 12-lead ECG, our study revealed that the prevalence of LAA thrombus was $6.7 \%$ with the most prevalent aetiology being paroxysmal AF. The presence of SEC can be a predictor of LAA thrombus in these patients.

\section{INTRODUCTION}

Ischaemic stroke is characterised as a neurological deficit caused by cerebral artery infarction leading to morbidity and mortality. There are various causes of this disease, such as cerebral large-artery atherosclerosis, cerebral small-vessel occlusion and cardioembolism. Identifying the aetiology of stroke is necessary to guide the therapeutic strategies for the treatment and prevention of recurrent stroke. ${ }^{1}$ Of the causes of ischaemic stroke, cardioembolism accounts for 14.6\%-36.0\%
Strengths and limitations of this study

- A major strength of our study is the prospective study setting.

- A limitation of this study is that it is based on data from a single centre.

- The impact of comorbidities on left atrial appendage thrombus is not fully evaluated.

of patients and occurs more frequently with increasing age. There are various underlying mechanisms and thrombi arising from the left atrial appendage (LAA) are the principal source of thromboembolism, predominantly in patients with atrial fibrillation (AF). ${ }^{2} \mathrm{LAA}$ thrombi can be detected using transoesophageal echocardiography (TEE), a reference standard imaging modality, with high specificity and sensitivity. ${ }^{3}$

The trabeculated LAA is a derivative of the primitive atrium located at the anterolateral portion of the smooth-walled atrial cavity. The LAA acts as a contractile reservoir and may modulate volume homoeostasis by the activation of stretch-sensitive receptors and by producing atrial natriuretic peptide. In $\mathrm{AF}$, the LAA is the prime location at which thrombus formation occurs resulting from chamber dilatation, endocardial dysfunction, increased thrombogenicity and decreased contractile ability. ${ }^{4}$ However, accumulating evidence has revealed that LAA thrombus may occur in patients with sinus rhythm through certain mechanisms, including left ventricular dysfunction, valvular heart diseases, atrial cardiopathy or subclinical AF. Two previous studies using TEE detected LAA thrombus in $6.2 \%$ and $9.3 \%$ of patients who had a stroke with sinus rhythm. ${ }^{56}$ Both studies excluded patients with history of AF or newly diagnosed AF on Holter monitoring; thus, paroxysmal AF could not be addressed. 
Furthermore, the participants in the two studies may not be fully representative of patients in clinical practice, because TEE was only performed in patients with strokes suspected to be cardioembolic in origin. The more recent one was a retrospective study; thus, there may have been selection bias. ${ }^{5}$

Therefore, we performed this cross-sectional study aimed at (1) investigating the prevalence of LAA thrombus detected by TEE in patients with acute ischaemic stroke (AIS) and sinus rhythm on 12-lead ECG, (2) screening for paroxysmal AF using 24-hour ECG monitoring and (3) identifying the predictors for LAA thrombus in these patients.

\section{METHODS}

\section{Sample size calculation}

Sample size was calculated for the first aim of this study using a single population proportion formula: $\mathrm{n}=\mathrm{Z}^{2}{ }_{1-\alpha / 2} *\left[\mathrm{p}^{*}(1-\mathrm{p}) / \mathrm{d}^{2}\right]$, with $\mathrm{Z}_{1-\alpha / 2}=1.96 \quad(\alpha=0.05 \%$ and 95\% CI) and $\mathrm{d}=$ =precision (assumed as 0.04 ). p=prevalence of LAA thrombus in patients who had a stroke with sinus rhythm (set as 9.3\%). The expected rate of exclusion related to patients having AF on 12-lead ECG was $10 \%$; therefore, the required sample size for this study was a minimum of 223 patients.

\section{Participant selection and data collection}

Between June 2019 and February 2021, a total of 349 patients admitted to our hospital within 48 hours following the onset of ischaemic stroke were prospectively referred to undergo TEE for the detection of LAA thrombus. We excluded 98 patients previously treated with thrombolytic therapy for the current stroke, 2 patients in whom the transoesophageal transducer could not be inserted, 3 patients with severe mitral stenosis, 21 patients with $\mathrm{AF}$ on 12-lead ECG and 2 patients who did not provide informed consent. The remaining 223 patients were included in the study. Written informed consent was provided by the patients or the patients' next of kin.

The ischaemic stroke divided according to the side of affected brain hemisphere (one side and both sides) which was demonstrated by brain MRI. Only new-onset infarcted regions were included in this study. CT angiography (CTA) was performed to detect atherosclerotic stenosis in the extracranial and intracranial arteries. Significant stenosis was considered as $\geq 70 \%$.

Transthoracic echocardiography (TTE) was performed prior to TEE with the patients in a partial left lateral decubitus position. The measurements of left atrium (LA) and left ventricular internal dimension in diastole (LVIDd) on parasternal long-axis, and biplane Simpson's left ventricular ejection fraction (LVEF) were based on previously established guidelines. ${ }^{7}$ We obtained mitral E/A ratio data based on the measurement of peak E-wave velocity and peak A-wave velocity. The E/A ratio was classified based on the recommendations of the American Society of Echocardiography and the European Association of
Cardiovascular Imaging for the evaluation of left ventricular diastolic function. ${ }^{8}$

The 12-lead ECG result on hospital admission was used as the baseline heart rhythm. After TEE, all patients underwent 24-hour ECG monitoring to detect underlying paroxysmal AF. The Holter monitor results were independently interpreted by two experienced cardiologists who were blinded to the TEE results. The demographic characteristics, comorbidity and biochemical analyses for all patients were obtained from interviews and electronic medical records.

\section{Transoesophageal echocardiographic examination}

TEE was performed in patients within 3 days of their admission to hospital. A $5 \mathrm{MHz}$ multiplane probe (Siemens Healthineers, Erlangen, Germany) was used in accordance with the European Association of Cardiovascular Imaging guidelines. ${ }^{9}$ The TEE evaluation of each patient was conducted by two experienced cardiologists who have performed $\geq 30$ TEE per month over a period of $>5$ five years. If there were any discrepancies, assessment by a third cardiologist was sought and the final decision was only made with the consensus of all three cardiologists.

LAA was visualised from the mid-oesophageal position at multiple omniplane angles. The LAA emptying velocity was obtained at an angle with the longest apex-to-orifice distance and the pulsed Doppler sample point was positioned at a depth of one-third from the LAA orifice. LAA length and LAA orifice diametre were measured in the view where the appendage was longest. LAA thrombus was diagnosed by the presence of a well-circumscribed echo-dense mass, present in multiplane throughout the cardiac cycle, and differentiated from pectinate muscles. ${ }^{10}$ Spontaneous echo contrast (SEC), an echogenic swirling pattern of blood flow, was assigned one of three grades: absent, mild or severe. Mild SEC was defined as a discrete echo contrast presenting in some parts of the LAA at high gain, whereas intensive echo contrast appearing throughout the entire LAA at normal gain was considered as severe. ${ }^{11}$ LAA morphology was classified into four types (cauliflower, cactus, chicken wing and windsock) according to the classifications of Wang and Kimura. ${ }^{12} 13$ Cactus and cauliflower morphologies have lengths $<40 \mathrm{~mm}$. Cactus has a dominant central lobe with secondary lobes, whereas cauliflower has complex internal structures. Chicken wing and windsock are longer than $40 \mathrm{~mm}$. Windsock has a dominant lobe with secondary lobes and a bend angle exceeding $100^{\circ}$, whereas chicken wing has only one lobe with a bend angle $<100^{\circ}$. Patent foramen ovale (PFO) was detected using colour Doppler TEE in the bicaval view with an angle of $80^{\circ}-110^{\circ}$. The other potential intracardiac sources of emboli were also screened on TEE.

\section{Statistical analysis}

All data were analysed using the IBM SPSS Statistics for Windows, V.130 25 (IBM Corp.). Qualitative data were described as frequencies and percentages 
Table 1 Baseline characteristics of patients based on the presence of thrombus

\begin{tabular}{|c|c|c|c|c|}
\hline Characteristics & $\begin{array}{l}\text { All patients } \\
(n=223)\end{array}$ & $\begin{array}{l}\text { Thrombus } \\
(n=15)\end{array}$ & $\begin{array}{l}\text { Non-thrombus } \\
(n=208)\end{array}$ & P value* \\
\hline Age, years & $66.2 \pm 11.3$ & $65.4 \pm 10.3$ & $66.2 \pm 11.4$ & 0.79 \\
\hline Age $\geq 65$ years, $n(\%)$ & $120(53.8)$ & $8(53.3)$ & $112(53.8)$ & 0.97 \\
\hline Male, $n(\%)$ & $137(61.4)$ & $8(53.3)$ & $129(62.0)$ & 0.51 \\
\hline AF in 24-hour ECG, n (\%) & $15(6.7)$ & 14 (93.3) & $1(0.5)$ & $<0.001$ \\
\hline Bilateral cerebral infarcts, $\mathrm{n}(\%)$ & $27(12.1)$ & $7(46.7)$ & $20(9.6)$ & $<0.001$ \\
\hline Stenosis $\geq 70 \%$ on CTA, $n$ (\%) & $169(75.8)$ & $7(46.7)$ & $162(77.9)$ & 0.02 \\
\hline \multicolumn{5}{|l|}{ Comorbidity, n (\%) } \\
\hline Dyslipidaemia & $173(77.6)$ & $14(93.3)$ & $159(76.4)$ & 0.20 \\
\hline Hypertension & $186(83.4)$ & $13(86.7)$ & $173(83.2)$ & 1.00 \\
\hline Coronary artery disease & $33(14.8)$ & $4(26.7)$ & $29(13.9)$ & 0.25 \\
\hline Heart failure & $3(1.3)$ & $0(0.0)$ & $3(1.4)$ & 1.00 \\
\hline Diabetes mellitus & $84(37.7)$ & $9(60.0)$ & $75(36.1)$ & 0.07 \\
\hline \multicolumn{5}{|l|}{ Laboratory findings } \\
\hline LDL-c, mmol/L & $3.2 \pm 1.1$ & $3.3 \pm 1.5$ & $3.2 \pm 1.1$ & 0.79 \\
\hline Non-HDL-c, mmol/L & $4.1 \pm 1.4$ & $4.2 \pm 1.8$ & $4.1 \pm 1.4$ & 0.75 \\
\hline Triglyceride, $\mathrm{mmol} / \mathrm{L}$ & $2.2 \pm 1.8$ & $2.0 \pm 1.2$ & $2.3 \pm 1.8$ & 0.57 \\
\hline Platelets, $\mathrm{K} / \mu \mathrm{L}$ & $243.6 \pm 65.1$ & $260.9 \pm 67.9$ & $242.4 \pm 64.9$ & 0.29 \\
\hline Mean platelet volume, $\mathrm{fL}$ & $7.9 \pm 1.2$ & $8.2 \pm 1.5$ & $7.9 \pm 1.2$ & 0.48 \\
\hline \multicolumn{5}{|l|}{ Transthoracic echocardiography } \\
\hline LVEF, \% & $68.0 \pm 8.0$ & $64.4 \pm 9.2$ & $68.2 \pm 7.8$ & 0.08 \\
\hline LVIDd, mm & $45.8 \pm 5.9$ & $47.9 \pm 6.1$ & $45.7 \pm 5.9$ & 0.15 \\
\hline LA, mm & $31.9 \pm 5.9$ & $33.0 \pm 4.5$ & $31.8 \pm 6.0$ & 0.43 \\
\hline Mitral E/A ratio, $n(\%)$ & & & & 0.59 \\
\hline$\leq 0.8$ & $93(41.7)$ & $7(46.7)$ & $86(41.3)$ & \\
\hline$>0.8$ to $<2$ & $100(44.8)$ & $5(33.3)$ & $95(45.7)$ & \\
\hline$\geq 2$ & $30(13.5)$ & $3(20.0)$ & 27 (13.0) & \\
\hline \multicolumn{5}{|c|}{ Valvular heart diseases (moderate), $\mathrm{n}(\%)$} \\
\hline Aortic regurgitation & $28(12.6)$ & $2(13.3)$ & $26(12.5)$ & 1.00 \\
\hline Mitral regurgitation & $24(10.8)$ & $3(20.0)$ & $21(10.1)$ & 0.21 \\
\hline Tricuspid regurgitation & $50(22.4)$ & $4(26.7)$ & $46(22.1)$ & 0.75 \\
\hline \multicolumn{5}{|c|}{ Antithrombotic therapy at discharge, $\mathrm{n}(\%)$} \\
\hline Aspirin plus clopidogrel & $207(92.8)$ & $0(0)$ & 207 (99.5) & $<0.001$ \\
\hline Dabigatran or rivaroxaban & $16(7.2)$ & $15(100)$ & $1(0.5)$ & $<0.001$ \\
\hline
\end{tabular}

Values are presented as the numbers of patients (\%), means \pm SD.

*Thrombus versus non-thrombus.

AF, atrial fibrillation; CTA, CT angiography; HDL-c, high density lipoprotein cholesterol; LA, left atrium; LDL-c, low-density lipoprotein cholesterol; LVEF, left ventricular ejection fraction; LVIDd, left ventricular internal dimension in diastole.

(\%). Quantitative data were described as means \pm SD. Comparisons were conducted using the $\chi^{2}$ test or Fisher's exact test for non-numerical data and Student's t-test for continuous variables. Univariate logistic regression was performed involving the potential risk factors for LAA thrombus formation. Variables with $\mathrm{p}<0.2$ in univariate analysis were selected for multivariate logistic regression. All variables were examined for interaction and multicollinearity. OR was shown with 95\% CI. Statistical significance was defined as a twotailed value of $\mathrm{p}<0.05$.

\section{Patient and public involvement}

No patients or public were involved in setting the research question or the outcome measures, nor were they involved in the design and implementation of the study. 
Table 2 Transoesophageal echocardiography findings of patients based on the presence of thrombus

\begin{tabular}{|c|c|c|c|c|}
\hline Characteristics & $\begin{array}{l}\text { All patients } \\
(n=223)\end{array}$ & $\begin{array}{l}\text { Thrombus } \\
(n=15)\end{array}$ & $\begin{array}{l}\text { Non-thrombus } \\
(n=208)\end{array}$ & P value* \\
\hline SEC, $n(\%)$ & $19(8.5)$ & $8(53.3)$ & $11(5.3)$ & $<0.001$ \\
\hline Severity of SEC, $n(\%)$ & & & & $<0.001$ \\
\hline Absent & $204(91.5)$ & $7(46.7)$ & $197(94.7)$ & \\
\hline Mild & $19(8.5)$ & $8(53.3)$ & $11(5.3)$ & \\
\hline Severe & $0(0.0)$ & $0(0.0)$ & $0(0.0)$ & \\
\hline Orifice size of LAA, mm & $15.9 \pm 3.9$ & $17.7 \pm 3.8$ & $15.8 \pm 3.9$ & 0.08 \\
\hline Length of LAA, mm & $29.3 \pm 7.0$ & $35.7 \pm 6.1$ & $28.8 \pm 6.9$ & 0.001 \\
\hline Morphology of LAA, n (\%) & & & & 0.03 \\
\hline Windsock & $19(8.5)$ & $4(26.7)$ & $15(7.2)$ & \\
\hline Chicken wing & $5(2.2)$ & $1(6.7)$ & $4(1.9)$ & \\
\hline Cauliflower & $31(13.9)$ & $2(13.3)$ & 29 (13.9) & \\
\hline Cactus & $168(75.3)$ & $8(53.3)$ & $160(76.9)$ & \\
\hline Flow velocity of $L A A, m / s$ & $0.90 \pm 0.2$ & $0.66 \pm 0.2$ & $0.92 \pm 0.2$ & 0.001 \\
\hline Patent foramen ovale, $\mathrm{n}(\%)$ & $20(9.0)$ & $1(6.7)$ & $19(9.1)$ & 1.00 \\
\hline
\end{tabular}

Values are presented as the numbers of patients (\%), means \pm SD.

*Thrombus versus non-thrombus.

LAA, left atrial appendage; SEC, spontaneous echo contrast.

\section{RESULTS}

\section{Prevalence of LAA thrombus and baseline characteristics of} patients

A total of 223 patients (mean age: $66.2 \pm 11.3$, range: $41-97$ years, men: $61.4 \%$ ) with AIS and sinus rhythm on 12-lead ECG were investigated. The TEE examination detected LAA thrombus in 15 patients $(6.7 \%)$. Of the patients with LAA thrombus, 14 patients $(93.3 \%)$ exhibited newly diagnosed paroxysmal AF on 24-hour ECG monitoring. The remaining patient was an older man with severe coronary artery disease (CAD) who did not exhibit AF during 24-hour ECG monitoring.

\section{Differences between the thrombus and non-thrombus groups}

Participants were assigned to thrombus and non-thrombus groups. Table 1 shows the baseline characteristics of all patients and the comparison between the groups. We observed no statistically significant differences in terms of clinical features and TTE findings between the groups, including age, sex, comorbidities (dyslipidaemia, hypertension, CAD and diabetes), laboratory findings (lipid profiles, platelet count and mean platelet volume) and TTE parameters (LA diametre, LVIDd, and LVEF). However, the thrombus group had a significantly higher rate of bilateral stroke than the non-thrombus group ( $46.7 \%$ vs $9.6 \%, \mathrm{p}<0.001)$.

TEE findings are shown in table 2. Compared with the non-thrombus group, the thrombus group exhibited a significantly higher rate of SEC ( $53.3 \%$ vs $5.3 \%, \mathrm{p}<0.001)$, longer LAA ( $35.7 \pm 6.1 \mathrm{~mm}$ vs $28.8 \pm 6.9 \mathrm{~mm}, \mathrm{p}=0.001)$ and lower peak LAA emptying velocity $(0.66 \pm 0.2 \mathrm{~cm} / \mathrm{s}$ vs $0.92 \pm 0.2 \mathrm{~cm} / \mathrm{s}, \mathrm{p}=0.001)$. No patient in this study exhibited severe SEC. PFO was identified in 19 patients in the non-thrombus, whereas only 1 patient in the thrombus group had PFO. However, there was no significant difference in the rate of PFO between the groups. Papillary fibroelastoma of the aortic valve was detected in 2 patients without LAA thrombus. Lambl's excrescences were observed in 3 patients with LAA thrombus and in 2 patients without LAA thrombus. There were no other valve vegetations or cardiac tumours detected by TEE. The most common LAA morphology in our study population was cactus, which was found in $75.3 \%$ of patients. The cauliflower, windsock and chicken wing morphologies were observed in $13.9,8.5$ and $2.2 \%$ of patients, respectively. The distribution of the LAA morphologies was significantly different between the groups $(p=0.03)$.

Our findings reveal that bilateral cerebral infarcts, SEC, longer LAA and lower peak LAA emptying velocity were more frequently reported in patients who had stroke with LAA thrombus.

\section{Determination of the potential risk factors for LAA thrombus}

Univariate and multivariate logistic regression analyses using the enter method were performed to identify predictors of LAA thrombus (table 3). In the unadjusted model, there were four factors associated with the presence of thrombi in the LAA: SEC, length of LAA, LAA morphology and flow velocity of LAA. In the adjusted model, only SEC (OR, 9.04; 95\% CI 2.12 to $38.54 ; p=0.003$ ) increased the probability of LAA thrombi being present.

\section{DISCUSSION}

LAA thrombi migrate to the brain causing cardioembolic stroke and result in significant morbidity and 
Table 3 Factors associated with left atrial appendage (LAA) thrombus in logistic regression analysis $(n=223)$

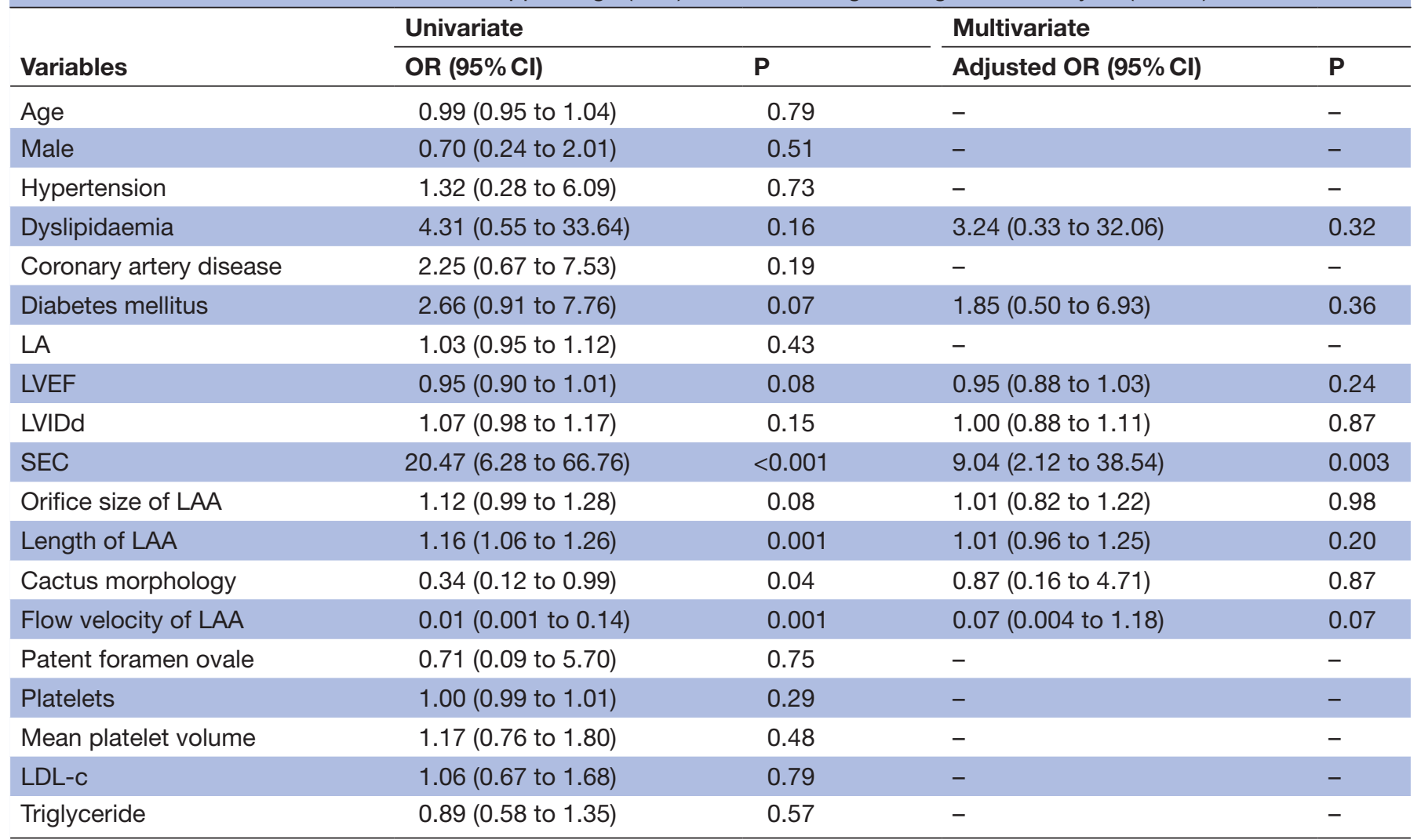

Variables that had a $p<0.2$ in univariate regression were entered into multiple regression.

LA, left atrium; LAA, left atrial appendage; LDL-c, low-density lipoprotein cholesterol; LVEF, left ventricular ejection fraction; LVIDd, left ventricular internal dimension in diastole; SEC, spontaneous echo contrast.

mortality. ${ }^{14}$ However, the underlying mechanism associated with LAA thrombus in patients who had a stroke with sinus rhythm on 12-lead ECG has not been elucidated and there are few reports regarding the prevalence of LAA thrombus in these patients. ${ }^{56}$ In the present study, we also observed evidence of LAA thrombus in patients who had a stroke with baseline sinus rhythm. In addition, SEC is a predictor and paroxysmal AF is the main cause of thrombus formation in LAA. To emphasise the importance of detecting the presence of LAA thrombus in patients who had a stroke, we propose five points for discussion.

\section{Presence of LAA thrombus in patients who had an ischaemic} stroke with sinus rhythm

Among the various causes of cardioembolic stroke, LAA thrombus is a treatable cause of cardiac embolism, and its detection can guide treatment with anticoagulant therapy. ${ }^{15}$ Although AF is the main cause of LAA thrombus formation, two previous studies reported the presence of LAA thrombus detected by TEE in patients who had a stroke without $\mathrm{AF}^{56}{ }^{5}$ Karabay et al used speckle tracking to evaluate LA deformations for predicting the presence of LAA thrombus in patients with suspected cardioembolic stroke. The prevalence of LAA thrombus in this study was $6.2 \%$ (9 of 144 patients). ${ }^{6}$ A higher rate $(9.3 \%, 14$ of 149 patients) of LAA thrombus was noted in the retrospective study by Cinar et $a l .{ }^{5}$ Our study identified LAA thrombus in 15 of 223 patients $(6.7 \%)$. These results suggest that it is reasonable to consider the presence of LAA thrombus in cases of newonset ischaemic stroke who exhibit normal sinus rhythm on 12-lead ECG.

\section{Presence of LAA thrombus in patients who had an ischaemic} stroke with atherosclerotic disease

When detected, the LAA thrombus is considered to be the cause of the stroke; however, determining the aetiology of ischaemic stroke is challenging when LAA thrombus and atherosclerotic disease coexist. Despite previous literature suggesting some distinct characteristics between cardioembolic and non-cardioembolic stroke, there is no gold standard to completely distinguish between the two events. ${ }^{14}$ Bearing this in mind, our study included all patients with and without atheroma on CTA, whereas the studies of Karabay et al and Cinar et al excluded patients who had a stroke if suspected to have atherothrombotic sources. ${ }^{56}$ We detected the presence of significant arterial disease in 7 of 15 patients with LAA thrombus (46.7\%), which indicates that LAA thrombus may coexist with atherosclerotic disease in patients who had an ischaemic stroke. 
Role of TEE in LAA thrombus detection in patients who had ischaemic stroke with sinus rhythm

TEE is commonly performed to identify cardioembolic sources. ${ }^{16}$ Despite a previous pharmacoeconomic analysis confirming the benefits of TEE for patients with new-onset ischaemic stroke who are in sinus rhythm, ${ }^{17}$ the routine use of this test for these patients is still debatable. ${ }^{18}$ First, although there are accumulating data revealing the presence of LAA thrombus in patients who had a stroke with sinus rhythm, ${ }^{56}$ in a study involving patients suffering from stroke and other systemic embolic events, LAA thrombus was not detected in any of the patients with sinus rhythm and a normal TTE examination. ${ }^{19}$ Second, in a meta-analysis (12 studies), the pooled rate of reported changes to anticoagulation therapy as a result of TEE findings among 3562 patients with AIS was $8.7 \% .^{20}$ However, some studies revealed only few patients who had an ischaemic stroke who experienced a change in management due to TEE findings. ${ }^{181}$ Further studies involving larger cohorts of patients who had an ischaemic stroke in sinus rhythm are required to determine a reasonable approach. Medical findings from clinical examination, laboratory tests and diagnostic imaging should be undertaken to evaluate which patients most require TEE examination.

\section{Aetiology of LAA thrombus formation in patients who had an ischaemic stroke with sinus rhythm}

$\mathrm{AF}$ is an independent risk factor for ischaemic stroke and patients with paroxysmal AF have an annual stroke rate similar to that of patients with chronic $\mathrm{AF}^{22}$ The cardioembolic stroke may be due to paroxysmal AF, which can be detected using an ECG Holter monitor. ${ }^{14}$ However, in two recent studies involving patients who had an ischaemic stroke with sinus rhythm, the role of paroxysmal $\mathrm{AF}$ as a potential cause of LAA thrombus was not assessed due to criteria exclusion. ${ }^{56}$ In our study, 15 of the 223 patients $(6.7 \%)$ experienced paroxysmal $\mathrm{AF}$, which was detected by 24-hour ECG monitoring. Our result is consistent with that of a meta-analysis of 50 studies showing that paroxysmal AF was newly diagnosed in $4.5 \%$ (95\% CI 2.7 to 6.7$)$ of patients after ischaemic stroke by in-hospital Holter monitoring. ${ }^{23}$ Notably, we detected paroxysmal AF in $93.3 \%$ of patients with LAA thrombus, whereas two previous studies reported that $75 \%-78 \%$ of patients who had a stroke with LAA thrombus had AF. ${ }^{24}{ }^{25}$ The higher rate of paroxysmal $\mathrm{AF}$ in our patients with LAA thrombus may be because none of our patients had been recently treated with antiarrhythmic drugs before enrolment whereas antiarrhythmic therapy was not an exclusion criterion in the two previous studies. ${ }^{24}{ }^{25}$ Our findings indicate that paroxysmal AF is likely the main aetiology predisposing LAA thrombus formation in patients who had an ischaemic stroke with sinus rhythm on 12-lead ECG and confirm the clinical benefit of screening for this arrhythmia in clinical practice.

The formation of LAA thrombus in paroxysmal AF is multifactorial and caused by complex pathophysiological mechanisms. LAA dysfunction is considered as a cause of thrombus formation ${ }^{26}$ and previous studies demonstrated that left atrial systolic myocardial dysfunction is represented as reduced left atrial strain and strain rate in speckle tracking echocardiography in patients with paroxysmal $\mathrm{AF}^{27-29}$ Importantly, the hypercoagulable state due to elevated levels of plasma von Willebrand factor, fibrinogen and P-selectin was observed in paroxysmal AF. 31 Moreover, left atrial electrophysiological remodelling may contribute to thrombogenesis because the prolonged atrial electromechanical interval may predict cardioembolic stroke in patients with paroxysmal $\mathrm{AF}^{32}$ Further study is required to elucidate how frequency, duration of paroxysmal $\mathrm{AF}$, atrial myopathy and underlying structural heart disease may influence LAA thrombus formation.

LAA thrombus was detected (figure 1) in the remaining older patient who did not experience AF during 24-hour ECG monitoring, but he had severe CAD. We assume that at least two causes contributed to LAA thrombus in the patient. First, the LAA thrombus is attributable to paroxysmal AF, but prolonged cardiac rhythm monitoring is required to increase the opportunity for detection of AF. ${ }^{33}$ Second, atrial myopathy caused by factors such as
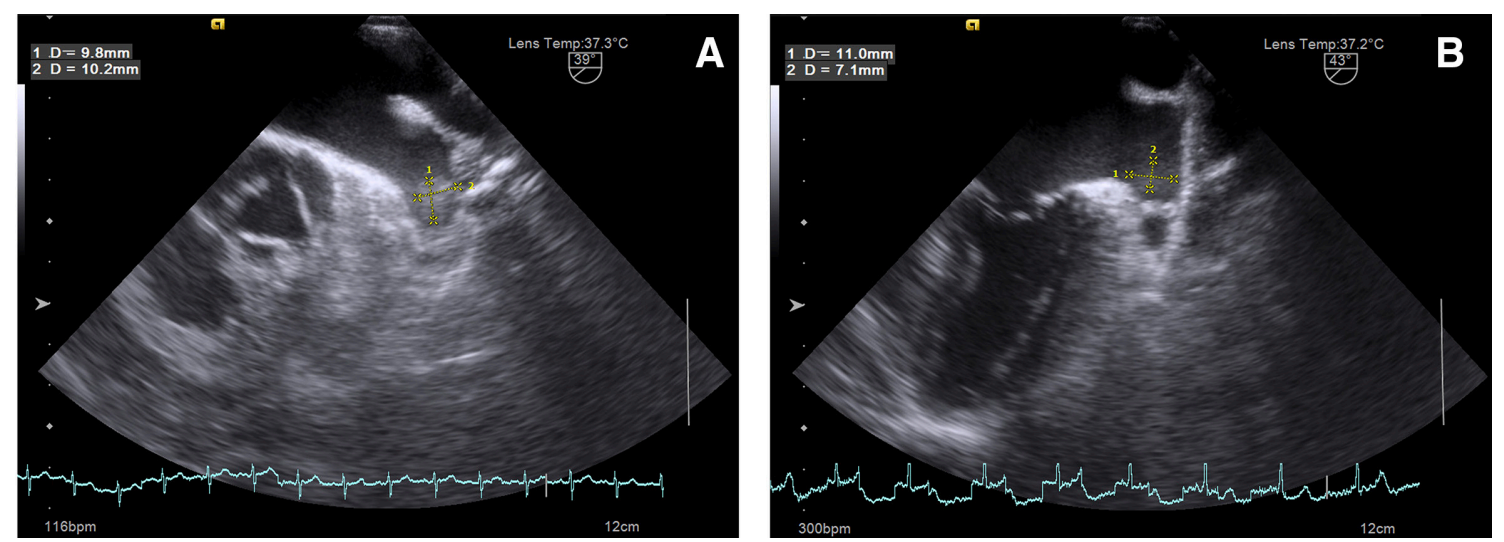

Figure 1 Left atrial appendage thrombus on transoesophageal echocardiography images in the two patients with sinus rhythm on 12-lead ECG. (A) This patient exhibited paroxysmal atrial fibrillation in 24-hour ECG monitoring. (B) An older patient had severe coronary artery disease with no prior intervention. 
ageing, myocardial ischaemia and electrophysiological remodelling may result in LAA thrombus independent of $\mathrm{AF}^{34} \mathrm{~A}$ longer follow-up and further medical examination are required to reveal the aetiology of thrombosis in the patient.

\section{Predictors of LAA thrombus in patients who had an ischaemic stroke with sinus rhythm}

Previous studies conducted on patients with AF have demonstrated that multiple-lobed LAA, reduced LAA flow velocity and SEC are risk factors for LAA thrombus, ${ }^{35-37}$ but there is conflicting evidence regarding a link between LAA morphology and the risk of thrombosis. ${ }^{38}{ }^{39}$ Limited information is available regarding the predictors of LAA thrombus in patients who had a stroke with baseline sinus rhythm. ${ }^{56}$ In such patients, while a recent study showed that mean platelet volume, renal function, LVEF and LAA flow velocity were independent predictors of LAA thrombus, ${ }^{5}$ another study revealed that LA peak strain and high-sensitivity $\mathrm{C}$ reactive protein were associated with LAA thrombus, but not mean platelet volume, renal function or LVEF.

In the current study, the adjusted model revealed that patients with SEC had a significantly higher probability of LAA thrombus formation. Although the LAA emptying velocity was lower and the LA dimension was greater in the thrombus group than in the non-thrombus group, they were not predictors of LAA thrombus formation in our study. Consistent with our findings, Fatkin et al demonstrated that SEC is the factor most strongly associated with LAA thrombus formation and cardioembolic events, ${ }^{37}$ and Sadanandan et al reported that SEC has a greater association with stroke risk than LAA flow velocity or atrial size in patients with sinus rhythm. ${ }^{40}$ However, mild SEC, a predictor of LAA thrombus in our study, can be inadequate as a clinical signal of the presence of thrombus on TEE. The first reason is that the prevalence of SEC varies based on types of AF, being the lowest among the patients with paroxysmal AF studied using TEE during sinus rhythm. ${ }^{37}$ Second, the dense swirling pattern of mild SEC may be difficult to recognise on TEE. These issues highlight the importance of careful examination of the LAA in multiple imaging planes to improve the diagnostic accuracy of SEC and LAA thrombi in patients with paroxysmal AF.

Patients with paroxysmal AF present distinct LAA flow velocity patterns based on the presence or absence of episodes of AF during TEE examination. ${ }^{37}$ Fatkin et al found that patients with paroxysmal AF studied using TEE during sinus rhythm had a nearly normal LAA emptying velocity $(0.57 \pm 0.3 \mathrm{~m} / \mathrm{s}$ vs $0.61 \pm 0.2 \mathrm{~m} / \mathrm{s}$ of controls with sinus rhythm), which was significantly higher than that in patients studied using TEE during episodes of $\mathrm{AF}^{37} \mathrm{In}$ agreement with the findings, all our patients with paroxysmal AF, the main aetiology predisposing patients to LAA thrombosis in our study, exhibited sinus rhythm during TEE, which may explain their reduced LAA emptying velocity $(0.69 \pm 0.2 \mathrm{~m} / \mathrm{s})$ (online supplemental table
S1) but not as much as that of patients with $\mathrm{AF}^{41}$ Since reduced LAA emptying velocity is significantly correlated with the formation of LAA thrombus, ${ }^{26}$ the thrombosis might predominantly occur in paroxysmal AF during episodes of AF than during sinus rhythm. Further studies are required to clarify the impact of heterogeneous characteristics of paroxysmal AF on the predictive role of LAA flow velocity in LAA thrombosis.

LA enlargement has been shown to be predictive of LAA thrombus in chronic $\mathrm{AF}^{42}{ }^{43}$ but its role in paroxysmal AF remains elusive. Fatkin et al found that LAA thrombi may present in patients with paroxysmal AF with a normal LA size. ${ }^{37}$ Two previous studies reported normal anteroposterior LA dimension in patients with paroxysmal AF with no significant changes in the LA size after a follow-up period of $20-30$ months. ${ }^{445}$ Further research is required to elucidate the association between LA size and the formation of LAA thrombus in patients who had an ischaemic stroke with sinus rhythm having paroxysmal AF

\section{Limitations}

This study has three main limitations. First, the LAA morphology obtained from two-dimensional TEE examination may not be reliable in terms of the complex structure of LAA. Second, ECG monitoring over a 24-hour period may be insufficient to detect underlying paroxysmal AF. Finally, the impact of comorbidities on LAA thrombus was not fully evaluated because of the low prevalence of CAD, heart failure and diabetes mellitus in the study. Therefore, further studies involving participants with more comorbid conditions, the use of realtime three-dimensional TEE and extending the duration of Holter ECG monitoring are required to confirm our conclusions.

\section{CONCLUSIONS}

TEE examination detected LAA thrombus in $6.7 \%$ of patients with AIS and sinus rhythm on 12-lead ECG. The presence of SEC is associated with a ninefold risk of LAA thrombus formation. In these patients, paroxysmal $\mathrm{AF}$ is the principal cause of LAA thrombus and should be a consideration for screening.

Acknowledgements We thank the patients for their participation in our study. Contributors HTN and HVBN designed the study. HTN, HVBN and HQL performed and analysed echocardiography measurements. HTN and HQN collected the clinical data, checked the validity of the data and performed the statistical analysis. HTN is responsible for the overall content as guarantor. HTN wrote the manuscript. All authors have read and approved the final manuscript.

Funding The authors have not declared a specific grant for this research from any funding agency in the public, commercial or not-for-profit sectors.

Competing interests None declared.

Patient consent for publication Not required.

Ethics approval Thong Nhat hospital, Ho Chi Minh city (57/2019/BVTN-HĐYĐ)

Provenance and peer review Not commissioned; externally peer reviewed.

Data sharing statement The datasets used and/or analysed during the current study are available from the corresponding author on reasonable request. Data are available upon reasonable request. 
Supplemental material This content has been supplied by the author(s). It has not been vetted by BMJ Publishing Group Limited (BMJ) and may not have been peer-reviewed. Any opinions or recommendations discussed are solely those of the author(s) and are not endorsed by BMJ. BMJ disclaims all liability and responsibility arising from any reliance placed on the content. Where the content includes any translated material, BMJ does not warrant the accuracy and reliability of the translations (including but not limited to local regulations, clinical guidelines, terminology, drug names and drug dosages), and is not responsible for any error and/or omissions arising from translation and adaptation or otherwise.

Open access This is an open access article distributed in accordance with the Creative Commons Attribution Non Commercial (CC BY-NC 4.0) license, which permits others to distribute, remix, adapt, build upon this work non-commercially, and license their derivative works on different terms, provided the original work is properly cited, appropriate credit is given, any changes made indicated, and the use is non-commercial. See: http://creativecommons.org/licenses/by-nc/4.0/.

\section{ORCID iD}

Huan Thanh Nguyen http://orcid.org/0000-0002-5086-7273

\section{REFERENCES}

1 Campbell BCV, De Silva DA, Macleod MR, et al. Ischaemic stroke. Nat Rev Dis Primers 2019;5:70.

2 Arboix A, Alió J. Cardioembolic stroke: clinical features, specific cardiac disorders and prognosis. Curr Cardiol Rev 2010;6:150-61.

3 Hwang JJ, Chen JJ, Lin SC, et al. Diagnostic accuracy of transesophageal echocardiography for detecting left atrial thrombi in patients with rheumatic heart disease having undergone mitral valve operations. Am J Cardiol 1993;72:677-81.

4 Naksuk N, Padmanabhan D, Yogeswaran V, et al. Left atrial appendage: embryology, anatomy, physiology, arrhythmia and therapeutic intervention. JACC Clin Electrophysiol 2016;2:403-12.

5 Cinar T, Hayiroğlu Mert İlker, Çiçek V, et al. Predictors of left atrial thrombus in acute ischemic stroke patients without atrial fibrillation: a single-center cross-sectional study. Rev Assoc Med Bras 2020;66:1437-43.

6 Karabay $\mathrm{CY}$, Zehir R, Güler A, et al. Left atrial deformation parameters predict left atrial appendage function and thrombus in patients in sinus rhythm with suspected cardioembolic stroke: a speckle tracking and transesophageal echocardiography study. Echocardiography 2013;30:572-81.

7 Mitchell C, Rahko PS, Blauwet LA, et al. Guidelines for performing a comprehensive transthoracic echocardiographic examination in adults: recommendations from the American Society of echocardiography. J Am Soc Echocardiogr 2019;32:1-64.

8 Nagueh SF, Smiseth OA, Appleton CP, et al. Recommendations for the Evaluation of Left Ventricular Diastolic Function by Echocardiography: An Update from the American Society of Echocardiography and the European Association of Cardiovascular Imaging. J Am Soc Echocardiogr 2016;29:277-314

9 Flachskampf FA, Wouters PF, Edvardsen T, et al. Recommendations for transoesophageal echocardiography: EACVI update 2014. Eur Heart J Cardiovasc Imaging 2014;15:353-65.

10 Seward JB, Khandheria BK, Oh JK, et al. Critical appraisal of transesophageal echocardiography: limitations, pitfalls, and complications. J Am Soc Echocardiogr 1992;5:288-305.

11 Daniel WG, Nellessen U, Schröder E, et al. Left atrial spontaneous echo contrast in mitral valve disease: an indicator for an increased thromboembolic risk. J Am Coll Cardiol 1988;11:1204-11.

12 Wang Y, Di Biase L, Horton RP, et al. Left atrial appendage studied by computed tomography to help planning for appendage closure device placement. J Cardiovasc Electrophysiol 2010;21:973-82.

13 Kimura T, Takatsuki S, Inagawa K, et al. Anatomical characteristics of the left atrial appendage in cardiogenic stroke with low CHADS2 scores. Heart Rhythm 2013;10:921-5.

14 Kamel H, Healey JS. Cardioembolic stroke. Circ Res 2017;120:514-26.

15 Powers WJ, Rabinstein AA, Ackerson T, et al. Guidelines for the early management of patients with acute ischemic stroke: 2019 update to the 2018 guidelines for the early management of acute ischemic stroke: a guideline for healthcare professionals from the American heart Association/American stroke association. Stroke 2019;50:e344-418.

16 Pepi M, Evangelista A, Nihoyannopoulos P, et al. Recommendations for echocardiography use in the diagnosis and management of cardiac sources of embolism: European association of echocardiography (EAE) (a registered branch of the ESC). Eur $J$ Echocardiogr 2010;11:461-76.

17 McNamara RL, Lima JA, Whelton PK, et al. Echocardiographic identification of cardiovascular sources of emboli to guide clinical management of stroke: a cost-effectiveness analysis. Ann Intern Med 1997;127:775-87.

18 Schwartz BG, Alexander CT, Grayburn PA, et al. Utility of routine transesophageal echocardiography in patients with stroke or transient ischemic attack. Proc 2018;31:401-3.

19 Leung DY, Black IW, Cranney GB, et al. Selection of patients for transesophageal echocardiography after stroke and systemic embolic events. Role of transthoracic echocardiography. Stroke 1995;26:1820-4.

20 Katsanos AH, Bhole R, Frogoudaki A, et al. The value of transesophageal echocardiography for embolic strokes of undetermined source. Neurology 2016;87:988-95.

21 Rosol ZP, Kopecky KF, Minehart BR, et al. Limitations of transoesophageal echocardiogram in acute ischaemic stroke. Open Heart 2020;7:e001176.

22 Hart RG, Pearce LA, Rothbart RM, et al. Stroke with intermittent atrial fibrillation: incidence and predictors during aspirin therapy. stroke prevention in atrial fibrillation Investigators. J Am Coll Cardiol 2000;35:183-7.

23 Sposato LA, Cipriano LE, Saposnik G, et al. Diagnosis of atrial fibrillation after stroke and transient ischaemic attack: a systematic review and meta-analysis. Lancet Neurol 2015;14:377-87.

24 Hur J, Kim YJ, Lee H-J, et al. Left atrial appendage thrombi in stroke patients: detection with two-phase cardiac CT angiography versus transesophageal echocardiography. Radiology 2009;251:683-90.

25 Senadeera SC, Palmer DG, Keenan R, et al. Left atrial appendage thrombus detected during hyperacute stroke imaging is associated with atrial fibrillation. Stroke 2020;51:3760-4.

26 Yaghi S, Song C, Gray WA, et al. Left atrial appendage function and stroke risk. Stroke 2015;46:3554-9.

27 Bukowska A, Hammwöhner M, Corradi D, et al. Atrial thrombogenesis in atrial fibrillation : Results from atrial fibrillation models and AF-patients. Herzschrittmacherther Elektrophysiol 2018;29:76-83.

28 Henein M, Zhao Y, Henein MY, et al. Disturbed left atrial mechanical function in paroxysmal atrial fibrillation: a speckle tracking study. Int J Cardiol 2012;155:437-41.

29 Kuppahally SS, Akoum N, Burgon NS, et al. Left atrial strain and strain rate in patients with paroxysmal and persistent atrial fibrillation: relationship to left atrial structural remodeling detected by delayed-enhancement MRI. Circ Cardiovasc Imaging 2010;3:231-9.

30 Li-Saw-Hee FL, Blann AD, Gurney D, et al. Plasma von Willebrand factor, fibrinogen and soluble P-selectin levels in paroxysmal, persistent and permanent atrial fibrillation. Effects of cardioversion and return of left atrial function. Eur Heart $J$ 2001;22:1741-7.

31 Hatzinikolaou-Kotsakou E, Kartasis Z, Tziakas D, et al. Atrial fibrillation and hypercoagulability: dependent on clinical factors or/ and on genetic alterations? J Thromb Thrombolysis 2003;16:155-61.

32 Hoshi Y, Nozawa Y, Ogasawara M, et al. Atrial electromechanical interval may predict cardioembolic stroke in apparently low risk elderly patients with paroxysmal atrial fibrillation. Echocardiography 2014;31:140-8.

33 Jones NR, Taylor CJ, Hobbs FDR, et al. Screening for atrial fibrillation: a call for evidence. Eur Heart J 2020;41:1075-85.

34 Shen MJ, Arora R, Jalife J. Atrial myopathy. JACC Basic Trans/ Sci 2019;4:640-54.

35 Yamamoto M, Seo Y, Kawamatsu N, et al. Complex left atrial appendage morphology and left atrial appendage thrombus formation in patients with atrial fibrillation. Circ Cardiovasc Imaging 2014;7:337-43

36 Goldman ME, Pearce LA, Hart RG, et al. Pathophysiologic correlates of thromboembolism in nonvalvular atrial fibrillation: I. Reduced flow velocity in the left atrial appendage (The Stroke Prevention in Atrial Fibrillation [SPAF-III] study). J Am Soc Echocardiogr 1999;12:1080-7.

37 Fatkin D, Kelly RP, Feneley MP. Relations between left atrial appendage blood flow velocity, spontaneous echocardiographic contrast and thromboembolic risk in vivo. J Am Coll Cardiol 1994;23:961-9.

38 Di Biase L, Santangeli P, Anselmino M, et al. Does the left atrial appendage morphology correlate with the risk of stroke in patients with atrial fibrillation? results from a multicenter study. J Am Coll Cardiol 2012;60:531-8.

39 Lupercio F, Carlos Ruiz J, Briceno DF, et al. Left atrial appendage morphology assessment for risk stratification of embolic stroke in patients with atrial fibrillation: a meta-analysis. Heart Rhythm 2016;13:1402-9. 
40 Sadanandan S, Sherrid MV. Clinical and echocardiographic characteristics of left atrial spontaneous echo contrast in sinus rhythm. J Am Coll Cardiol 2000;35:1932-8.

41 Agmon Y, Khandheria BK, Gentile F, et al. Echocardiographic assessment of the left atrial appendage. J Am Coll Cardiol 1999;34:1867-77.

42 Doukky R, Khandelwal A, Garcia-Sayan E, et al. External validation of a novel transthoracic echocardiographic tool in predicting left atrial appendage thrombus formation in patients with nonvalvular atrial fibrillation. Eur Heart J Cardiovasc Imaging 2013;14:876-81.
43 Ayirala S, Kumar S, O'Sullivan DM, et al. Echocardiographic predictors of left atrial appendage thrombus formation. J Am Soc Echocardiogr 2011;24:499-505.

44 Villecco AS, Pilati G, Bianchi G, et al. Left atrial size in paroxysmal atrial fibrillation: echocardiographic evaluation and follow-up. Cardiology 1992;80:89-93.

45 Rostagno C, Olivo G, Comeglio M, et al. Left atrial size changes in patients with paroxysmal lone atrial fibrillation. An echocardiographic follow-up. Angiology 1996;47:797-801. 